\title{
Residual active granzyme B in cathepsin C-null lymphocytes is sufficient for perforin-dependent target cell apoptosis
}

\author{
Vivien R. Sutton, ${ }^{1}$ Nigel J. Waterhouse, ${ }^{1}$ Kylie A. Browne, ${ }^{1}$ Karin Sedelies, ${ }^{1}$ Annette Ciccone, , Desiree Anthony, \\ Aulikki Koskinen, ${ }^{2}$ Arno Mullbacher, ${ }^{2}$ and Joseph A. Trapani ${ }^{1}$ \\ 'Cancer Immunology Program, Research Division, Peter MacCallum Cancer Centre, East Melbourne, VIC 3002, Australia \\ ${ }^{2}$ Viral Immunology, Division of Immunology and Genetics, John Curtin School of Medical Research, The Australian National University, Canberra, ACT O200, Australia
}

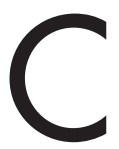
athepsin $\mathrm{C}$ activates serine proteases expressed in hematopoietic cells by cleaving an $\mathrm{N}$-terminal dipeptide from the proenzyme upon granule packaging. The lymphocytes of cathepsin C-null mice are therefore proposed to totally lack granzyme B activity and perforin-dependent cytotoxicity. Surprisingly, we show, using live cell microscopy and other methodologies, that cells targeted by allogenic CD8 ${ }^{+}$cytotoxic $T$ lymphocyte $(\mathrm{CTL})$ raised in cathepsin $\mathrm{C}$-null mice die through perforindependent apoptosis indistinguishable from that induced by wild-type CTL. The cathepsin C-null CTL expressed

\section{Introduction}

The granzymes, a family of structurally related serine proteases expressed in cytotoxic lymphocytes, cooperatively bring about the death of transformed and virus-infected cells after their cosecretion with perforin (Trapani and Smyth, 2002). Perforin is critical for permitting the access of granzymes and other granule-bound toxins to their substrates within the target cell (Froelich et al., 1996; Browne et al., 1999), and as a result, all granzyme-dependent cell death pathways are halted in its absence, leading to marked immunodeficiency in humans (Stepp et al., 1999; Voskoboinik et al., 2004) and mice (Kagi et al., 1994). By comparison, deficiency of an individual granzyme is better tolerated by gene-targeted mice. The absence of granzyme B (GrB), which cleaves target cell proteins adjacent to aspartate residues, results in delayed target cell DNA fragmentation during apoptosis of most, but not all, cell types (Heusel et al., 1994; Simon et al., 1997; Pardo et al., 2002). GrA induces a

Correspondence to Joseph A. Trapani: joe.trapani@petermac.org

Abbreviations used in this paper: AV, annexin $\mathrm{V} ; \mathrm{BLT}, \mathrm{N}^{\mathrm{a}}$-CBZ-L-lysine thiobenzyl ester; Cat, cathepsin; CTL, cytotoxic T lymphocyte; ECTV, ectromelia virus; NE, neutrophil elastase; NK, natural killer; PI, propidium iodide; PLS, PapillonLefevre syndrome.

The online version of this article contains supplemental material. reduced but still appreciable granzyme B activity, but minimal granzyme $A$ activity. Also, in contrast to mice with inactivation of both their granzyme $A / B$ genes, cathepsin $C$ deficiency did not confer susceptibility to ectromelia virus infection in vivo. Overall, our results indicate that although cathepsin C clearly generates the majority of granzyme B activity, some is still generated in its absence, pointing to alternative mechanisms for granzyme B processing and activation. Cathepsin $C$ deficiency also results in considerably milder immune deficiency than perforin or granzyme A/B deficiency. caspase-independent form of cell death that involves the induction of single-stranded DNA nicks after cleavage and activation of constituents of the SET complex (Lieberman and Fan, 2003). Proapoptotic function has also more recently been described for GrC (Johnson et al., 2003) and GrM (Kelly et al., 2004; Lu et al., 2006).

All of the granzymes and the closely related myeloid serine proteases, such as cathepsin $\mathrm{G}(\mathrm{CatG})$, are members of the chymotrypsin superfamily and, like chymotrypsin, are synthesized as preproenzymes (Salvesen and Enghild, 1990; Dikov et al., 1994). The signal peptide is cleaved by a signal peptidase in the endoplasmic reticulum, before transport to the granules (Caputo et al., 1993; Dikov et al., 1994). The proteases are then stored within the granules as active enzymes after further limited proteolysis that removes a two-amino-acid activation peptide at their $\mathrm{N}$ terminus (Jenne et al., 1988a). This enables the protease to assume the correct conformation for access and hydrolysis of substrate (Salvesen and Enghild, 1990). A similar process is used in the activation of the mast cell proteases, which are also stored as active enzymes in the lysosomal compartment (Salvesen and Enghild, 1990; Dikov et al., 1994). Inhibition of a specific dipeptidyl peptidase, CatC (dipeptidylpeptidase I) can 
prevent this processing and activation (McGuire et al., 1993). Transfection of mammalian COS-7 (Caputo et al., 1993; Smyth et al., 1995) or yeast (Pham et al., 1998) cells with granzyme cDNA constructs that retained the activation dipeptide resulted in the expression of inactive protease. However, the activity could be rescued by exogenous treatment of cell lysates or purified granzyme protein with CatC (Kummer et al., 1996; Pham et al., 1998), by coexpression of progranzyme and active CatC in the same cells or by deleting the dipeptide sequence before transfection (Caputo et al., 1993; Smyth et al., 1995).

Further evidence for a critical role of CatC in the processing of granzymes and myeloid cell serine proteases came from gene-knockout studies, in that a CatC-deficient mouse was generated by homologous recombination with an inactive CatC gene (Pham and Ley, 1999). Several studies have examined the effect of CatC deletion on the activity both of the granzymes and the structurally related mast cell and neutrophil proteases (Pham and Ley, 1999; Wolters et al., 2001; Adkison et al., 2002). Effector lymphocytes generated from these mice were reported to be as deficient as perforin-null mice in their ability to induce apoptosis of target cells, although only DNA fragmentation was assayed in these studies (Pham and Ley, 1999; Podack, 1999). No tryptase and only minimal ASPase activity could be detected in the granules isolated from CatC-null lymphocytes (Pham and Ley, 1999; Podack, 1999). Thus, it appeared that CatC deficiency effectively recapitulated the GrAB-null phenotype in cytotoxic lymphocytes and would also result in minimal activity from the other granzymes.

Given the proposed generic role for CatC in granzyme activation, we reasoned that cytotoxic T lymphocyte (CTL) from $\mathrm{CatC}^{-/-}$mice might allow us to study the physiological role of perforin in a setting where all of the killer cell granzymes were not expressed. Also, as perforin is present in CTLs of both $\mathrm{GrAB}^{-/-}$and $\mathrm{CatC}^{-1-}$ mice, comparing these CTLs would also allow us to determine the physiological role of the remaining "orphan" granzymes expressed in the $\mathrm{GrAB}^{-/-}$mice. Surprisingly, we found low but clearly measurable residual GrB activity in the absence of CatC expression, and that effector lymphocytes from CatC-null mice could induce apoptosis of target cells by a mechanism that was indistinguishable from classic apoptosis. Unlike $\mathrm{GrAB}^{-/-}$mice, which are exquisitely sensitive to infection with ectromelia virus (ECTV; mouse pox), CatC-deficient effector lymphocytes also mediated resistance to ECTV in vivo, so that infected mice were as resistant to the virus as wild-type C57BL/6 (B6) mice.

\section{Results}

\section{CatC-deficient CTLs induce target cell death through apoptosis}

It has previously been reported that $\mathrm{CatC}^{-1-}$ gene-targeted mice lack GrA and -B activities and therefore have severe defects of granule-mediated lymphocytotoxicity (Pham and Ley, 1999; Podack, 1999). To explore this issue further, we generated alloreactive CTLs in standard, one-way $\left(\mathrm{H}-2^{\mathrm{b}}\right.$ anti- $\left.\mathrm{H}-2^{\mathrm{k}}\right)$, mixed lymphocyte reactions and assessed the capacity of B6.CatC ${ }^{-1-}$ effector cells to kill mouse MS9II (H-2 $\left.{ }^{k}\right)$ target cells. We had previously used time-lapse microscopy at $37^{\circ} \mathrm{C}$ to characterize in real time the morphological and molecular events accompanying the death of MS9II target cells in response to alloreactive CTLs raised in $\mathrm{B} 6$ or $\mathrm{B} 6 . \mathrm{GrAB}^{-1-}$ mice (Waterhouse et al., 2006). This methodology allowed us to examine changes in cell morphology and the appearance of classic markers of apoptosis in response to CTL attack. By adding soluble annexin V (AV) and propidium iodide (PI) to the medium, we were able to track phosphatidylserine exteriorization (a relatively early marker of apoptosis) and loss of plasma membrane integrity (a late marker), respectively, as a function of time. Both B6 and B6.GrAB ${ }^{-1-}$ CTLs induced classic apoptotic changes, such as cell shrinkage, marked membrane blebbing, and nuclear collapse with indistinguishable kinetics (Waterhouse et al., 2006). However, a major point of difference was that although B6 CTLs induced early and marked target cell AV binding, cells killed by $\mathrm{GrAB}^{-1-}$ CTLs did not stain with AV until very late, simultaneously with loss of plasma membrane integrity (Waterhouse et al., 2006).

Given that, like $\mathrm{GrAB}^{-/-}$CTLs, CatC ${ }^{-/-}$CTLs had also been found to lack GrA or -B activity (Pham and Ley, 1999), we were surprised to find that MS9II cells exposed to $\mathrm{CatC}^{-1-}$ CTLs underwent morphological and molecular events of apoptosis that were indistinguishable from B6 CTLs, both in their amplitude and timing. B6 or B6.CatC ${ }^{-1-}$ effector cells were added to equal numbers of the much larger and adherent MS9II target cells. The time course of death in a single representative target cell is shown (Fig. $1 \mathrm{~A}$ ). In this cell, rounding occurred at $\sim 1 \mathrm{~h} 10 \mathrm{~min}$, followed by AV binding at $1 \mathrm{~h} 45 \mathrm{~min}$ and PI uptake at $2 \mathrm{~h} 25 \mathrm{~min}$. Fig. $1 \mathrm{~B}$ and Video 1, available at http://www.jcb.org/cgi/content/full/jcb.200609077/DC1, illustrate the sequence of events for the same cell, including conjugate formation with the CTL $(\mathrm{t}=0.5 \mathrm{~h})$, cell rounding and plasma membrane blebbing $(\mathrm{t}=1.5 \mathrm{~h}), \mathrm{AV}$ binding $(\mathrm{t}=2.0 \mathrm{~h})$, and PI uptake $(\mathrm{t}=2.5 \mathrm{~h})$. By making similar observations on a large number of dying target cells, it was possible to compare both the morphology and kinetics of death induced by CatC-deficient and -sufficient CTLs (Fig. $1 \mathrm{C}$ ) with those raised in $\mathrm{GrAB}^{-/-}$ mice (Waterhouse et al., 2006). Taking the time of rounding as a common reference point (i.e., $\mathrm{t}=0$ ), no difference was observed in the kinetics of cell death (morphological changes; AV or PI staining) over populations of cells killed by B6 CTLs $(n=20)$ or B6.CatC ${ }^{-/-}$CTLs $(n=25)$. The killing observed in these assays was mediated though the granule pathway in that CTLs raised simultaneously in perforin-deficient mice were unable to kill the same target cells, as demonstrated both by time-lapse microscopy (not depicted) or $4-\mathrm{h}^{51} \mathrm{Cr}$ release assays, whereas target cells released ${ }^{51} \mathrm{Cr}$ in response to both wild-type and $\mathrm{CatC}^{-/-}$CTLs (Fig. 1 D). Cell death by perforin-induced lysis was excluded, as the kinetics and morphology of this form of cell death are quite easily distinguished from apoptosis (Waterhouse et al., 2006). We also demonstrated by Western blot that the $\mathrm{CatC}^{-/-}$mice had not up-regulated their level of perforin or GrB protein expression compared with B6 CTLs (Fig. 1, $\mathrm{E}$ and F). In total, these observations strongly suggested that the CatC-deficient effector cells induce apoptotic death similar to wild-type B6 killer cells, but differing from cell death induced 

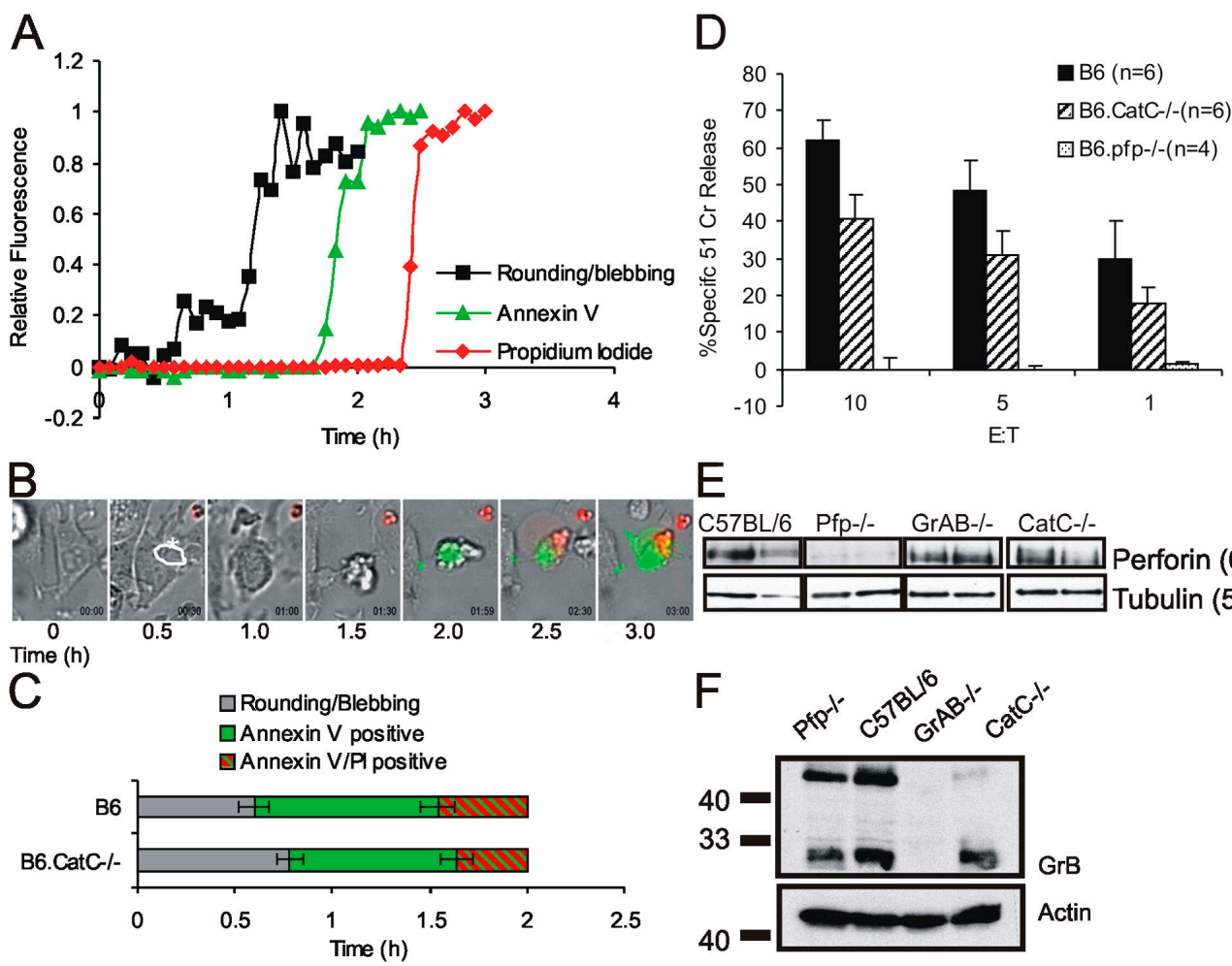

$\mathrm{E}$
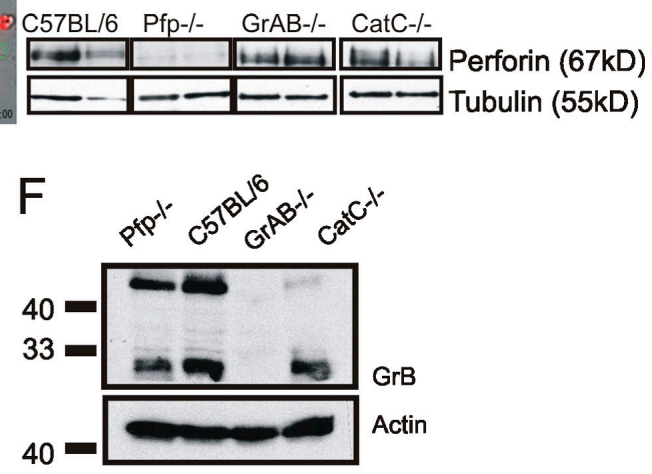

Figure 1. $\mathrm{CatC}^{-/-} \mathrm{CD}^{+}$CTLs induce apoptosis in target cells. (A) Kinetics of apoptotic events in a representative MS9II target cell after exposure to BL/6. $\mathrm{CatC}^{-/-}$effector lymphocytes $\left(>90 \% \mathrm{CD} 8^{+}\right)$generated from day 3 secondary-stimulated mixed lymphocyte reaction $\left(\mathrm{H}-2^{\mathrm{b}}\right.$ anti $\left.\mathrm{H}-2^{\mathrm{k}}\right)$. Phosphatidylserine exposure was measured by the fluorescence of AV binding, and loss of membrane integrity was measured by PI uptake, relative to maximum fluorescence. In these experiments, $t=0$ was taken as the time at which target cell rounding occurred. There was no difference in the cumulative number of target cells becoming rounded, either in the presence or absence of CatC expression. Images were recorded every $5 \mathrm{~min}$. (B) The morphology of the cell in A is shown in the series of photographs. $\mathrm{T}=0 \mathrm{~h}$ shows the untreated target cell, $\mathrm{t}=0.5 \mathrm{~h}$ shows CTL-target cell conjugation (CTL is enclosed by a white line and marked with an asterisk), and $t=1.5 \mathrm{~h}$ shows the round/blebbing target cell. AV binding is clearly evident at $t=2.0 \mathrm{~h}$, and PI uptake is evident by $t=$ 2.5 h. See Video 1 (available at http://www.jcb.org/cgi/content/full/jcb.200609077/DC1). (C) The duration from rounding to AV binding and AV binding to PI uptake was calculated for individual target cells incubated with either B6 or B6. CatC ${ }^{-1-}$ CTL. Mean \pm SEM ( $n=20$ for B6 treated cells, and $n=25$ for B6.CatC ${ }^{-1-}$ treated cells). (D) BL/6, BL/6. CatC ${ }^{-1-}$, and B6.pfp ${ }^{-/-}$effector lymphocytes $\left(\mathrm{H}-2^{\mathrm{b}}\right.$ anti $\mathrm{H}-2^{\mathrm{k}}$; $\left.>90 \% \mathrm{CD}^{+}\right)$were incubated with ${ }^{51} \mathrm{Cr}$-labeled MS9II target cells in a 4-h assay at the effector/target ratios indicated. In each case, the data points show the mean of independent experiments (each of which was performed in triplicate) \pm SEM. The number of independent experiments performed for each effector type is indicated in the figure. (E) Western blot analysis showing the presence of perforin using the rat monoclonal antibody PI-8 in the lysates of activated T cells from the various mouse strains indicated. Two pools of two mice (shown in separate lanes) were used for each strain. Blot was reprobed with an anti-tubulin antibody. (F) Western blot analysis showing the presence of GrB in the lysates of activated T cells from the various mouse strains indicated. Blot was reprobed with an anti-actin antibody.

by CTLs raised from mice with structural disruption of both their GrA and -B genes.

To exclude the possibility that the aforementioned findings were peculiar to MS9II target cells or the specific strain combination used to raise allo-reactive CTLs, we exposed ${ }^{51} \mathrm{Cr}$ labeled P815 mouse mastocytoma tumor cells $\left(\mathrm{H}-2^{\mathrm{d}}\right)$ to B6 CTLs stimulated with irradiated BALB/c splenocytes. Unlike MS9II cells (which have a relatively long doubling time), the DNA of rapidly dividing P815 cells labels well with ${ }^{125} \mathrm{I}-\mathrm{UdR}$, so that cells doubly labeled with ${ }^{51} \mathrm{Cr}$ and ${ }^{125} \mathrm{I}$ can be used to simultaneously evaluate plasma membrane permeability and DNA fragmentation. Both day 7 primary (Fig. 2 A) and day 3 secondary (Fig. 2 B) CTLs of B6 mice produced strong ${ }^{51} \mathrm{Cr}$ and ${ }^{125}$ I-DNA release that was only slightly diminished when CTLs from B6.CatC ${ }^{-l-}$ mice were used. This killing was once again mediated through the granule pathway, as doubly stimulated perforin-deficient CTLs induced neither ${ }^{51} \mathrm{Cr}$ or ${ }^{125} \mathrm{I}$ release, whereas the cell death induced by day 7 primary B6 CTLs was totally inhibited when free calcium in the reaction medium was complexed with EGTA (Fig. 2 and Fig. S1, available at http:// www.jcb.org/cgi/content/full/jcb.200609077/DC1).

Additional effector cell types were examined to determine whether the cytotoxic activity observed in allo-stimulated CatC-null CTLs was also seen in other effector lymphocytes. Unfractionated splenic natural killer $(\mathrm{NK})$ cells or purified $\mathrm{DX} 5^{+} \mathrm{CD}{ }^{-}$cells activated with $\mathrm{IL}-2$ each induced ${ }^{51} \mathrm{Cr}$ release from Yac-1 target cells in a perforin-dependent manner, but this did not vary whether CatC was expressed or not (unpublished data).

\section{Granule serine protease activity in CatC C $^{-1-}$ Iymphocytes}

Although perforin and granzymes synergistically bring about target cell apoptosis, it is well recognized that purified perforin is unable to induce DNA fragmentation, but only causes cell lysis (Duke et al., 1989). When delivered by a CTL or NK cell, GrA and -B activate separate signaling pathways, resulting in DNA nicking or oligonucleosomal fragmentation, respectively, in a perforin-dependent manner (Shi et al., 1992; Beresford 
A
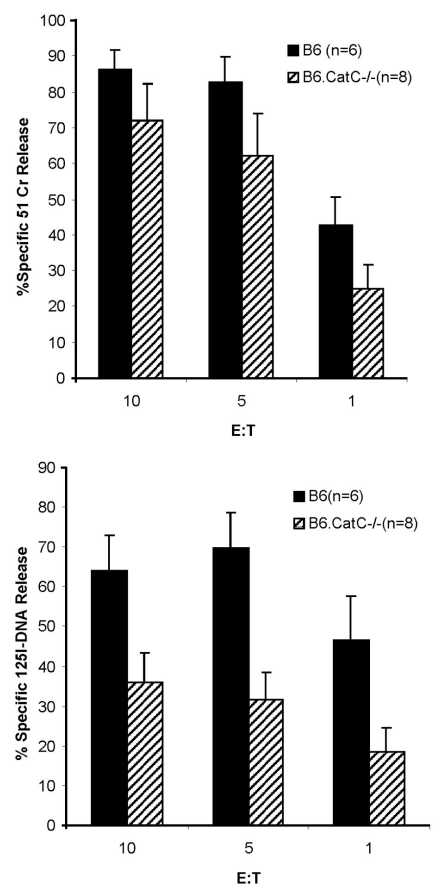

B
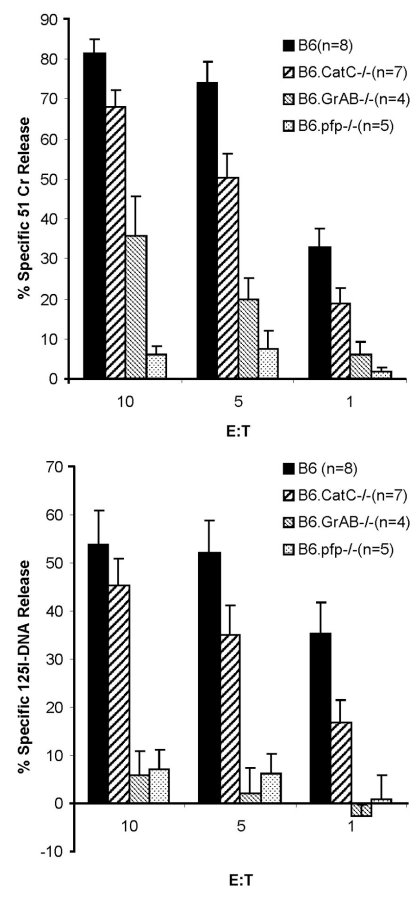

Figure 2. CatC $-/-$ CTLs induce both plasma membrane damage and DNA fragmentation in target cells. ${ }^{51} \mathrm{Cr}$ release and ${ }^{125}$-DNA release from target cells were used as measures of membrane damage and DNA fragmentation, respectively. ${ }^{51} \mathrm{Cr}$ - and ${ }^{125} \mathrm{I}-\mathrm{UdR}$-labeled P815 mastocytoma cells were coincubated for $4 \mathrm{~h}$ with day 7 primary stimulated (A) or day 3 secondarily stimulated (B) effector cells $\left(\mathrm{H}-2^{\mathrm{b}}\right.$ anti-H-2d) from $\mathrm{BL} / 6$ and $\mathrm{BL} / 6$. CatC ${ }^{-1}$ mice. $\mathrm{BL} / 6 . \mathrm{GAB}^{-1-}$ and $\mathrm{BL} / 6 . \mathrm{pfp}^{-1-}$ effectors were also examined in $\mathrm{B}$. The data points show the mean of independent experiments (each of which was performed in triplicate) \pm SEM. The number of independent experiments performed for each effector type is indicated in the figure. E:T, effector/ target ratio.

et al., 1999). Rapid fragmentation of target cell DNA, a hallmark of apoptosis induced through the granule-exocytosis mechanism, in most cell types is largely due to the action of active GrB (Heusel et al., 1994; Trapani et al., 1998a,b; Pardo et al., 2002). Consistent with these previous findings, we found that DNA fragmentation was abolished in P815 target cells exposed to $\mathrm{GrAB}^{-1-} \mathrm{CTLs}$, whereas appreciable ${ }^{51} \mathrm{Cr}$ release was still observed, as previously reported (Simon et al., 1997; Waterhouse et al., 2006; Fig. 2). In contrast, CatC-null CTLs were efficiently able to induce DNA fragmentation of P815 cells (Fig. 2). Although an earlier study failed to find evidence of GrA or -B activity in the lymphocytes of CatC-null mice (Pham and Ley, 1999), the similar kinetics of the apoptotic events detailed in CatC-sufficient and -deficient CTLs (Fig. 1) suggested that some serine protease (i.e., granzyme) activities might be generated in allo-stimulated, CatC-deficient CTLs. In addition to activating granzymes, CatC has been reported to be critical for activation of serine proteases in monocytes/neutrophils (Adkison et al., 2002) and mast cells (Wolters et al., 2001). To show that inactivation of the $\mathrm{CatC}$ gene abolished the activity of certain specific serine proteases in myeloid cells and to reconfirm the myeloid cell phenotype of the $\mathrm{CatC}^{-/-}$mice, $\mathrm{CatG}$ and neutrophil elastase (NE) were assayed in bone marrow neutrophils purified from mice administered granulocyte colony-stimulating factor, as described previously (Adkison et al., 2002). The hydrolysis of NE- and CatG-specific substrates was measured in a chromogenic assay using lysates of bone marrow cells from wild-type B6 and B6.CatC ${ }^{-1-}$ mice. As expected (Adkison et al., 2002), the activity of both enzymes was virtually abolished (reduced by $>98 \%$ ) in the absence of CatC expression (Fig. S2, available at http://www.jcb.org/cgi/content/full/jcb.200609077/DC1).

To test the possibility that the CTLs of $\mathrm{CatC}^{-/-}$mice were still capable of some residual granzyme activation, lysates prepared from allo-stimulated primary and secondary CD ${ }^{+}$CTLs ( $>90 \%$ pure) were assayed for their capacity to hydrolyze the GrB-specific substrate Ala-Ala-Asp-S-benzyl (cleavage after asp or ASPase activity), $\mathrm{N}^{\mathrm{a}}$-CBZ-L-lysine thiobenzyl ester (BLT; to measure trypsin-like GrA [tryptase] activity) and PheLeu-Phe-S-Benzyl (chymotrypsin-like or chymase activity; Fig. 3). It became clear that although GrB activity was markedly reduced, it was not abolished in the $\mathrm{CatC}^{-1-}$ cells. In kinetic assays, ASPase activity was reduced but still clearly detectable in the lysate of day 7 primary (Fig. $3 \mathrm{~A}$ ) or day $3 \mathrm{sec}$ ondary (Fig. 3 D) allo-stimulated CatC-null CTLs, albeit with some delay compared with CatC-sufficient cells. Not surprisingly, there was greater ASPase activity in twice-stimulated $\mathrm{CD}^{+}$cells; however, maximum substrate hydrolysis was reached by $10 \mathrm{~min}$ in both cases. The ASPase substrate, AlaAla-Asp-SBzl is cleaved only by GrB: it is not cleaved by caspases, proapoptotic cysteine proteases that also cleave after specific aspartate residues. We also performed an additional important control to examine the reliance of substrate turnover on the presence of bona fide granzyme activity: we demonstrated that no ASPase activity whatsoever was present in the CTLs of GrAB-null cells, in which the GrB gene is structurally disrupted (Fig. 3, A, D, and E). This observation ruled out any incidental cleavage by proteases other than $\mathrm{GrB}$ as a cause of substrate hydrolysis in the CatC-null cells. The presence of ASPase activity in the CatC-null lysate was again confirmed when lysates containing equal amounts of protein from one of the experiments shown in Fig. 3 D were progressively diluted and the assay was performed for $15 \mathrm{~min}$. At each lysate dilution, the level of ASPase activity was about one third of that seen in B6 lysates (Fig. 3 E). As expected, normal activity was noted in the B6.pfp ${ }^{-1-}$ lysates. As the substrate used in these assays is specific for $\mathrm{GrB}$, these results indicate that a proportion of the $\mathrm{GrB}$ precursor expressed in the CatC-null effectors had been activated. In contrast to ASPase activity, no measurable tryptase (GrA) activity was detected in either the CatC-null or $\mathrm{GrAB}^{-1-}$ lysates, indicating that $\mathrm{GrA}$ (and the second tryptase, $\mathrm{GrK}$ ) were not activated in the $\mathrm{CatC}^{-/-}$CTLs (Fig. 3, B and F). As expected, the turnover of the chymase substrate in the same lysates was not reduced in any of the lysates tested (Fig. 3, C and G). This indicated that $\mathrm{CatC}$ is not essential for the processing of at least some of the granzymes with chymase activity, presumably GrC-F (Trapani, 1998).

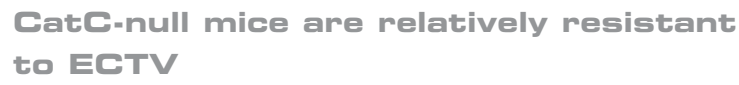

It was previously demonstrated that normally resistant B6 mice lacking both GrA and -B or perforin are incapable of controlling 

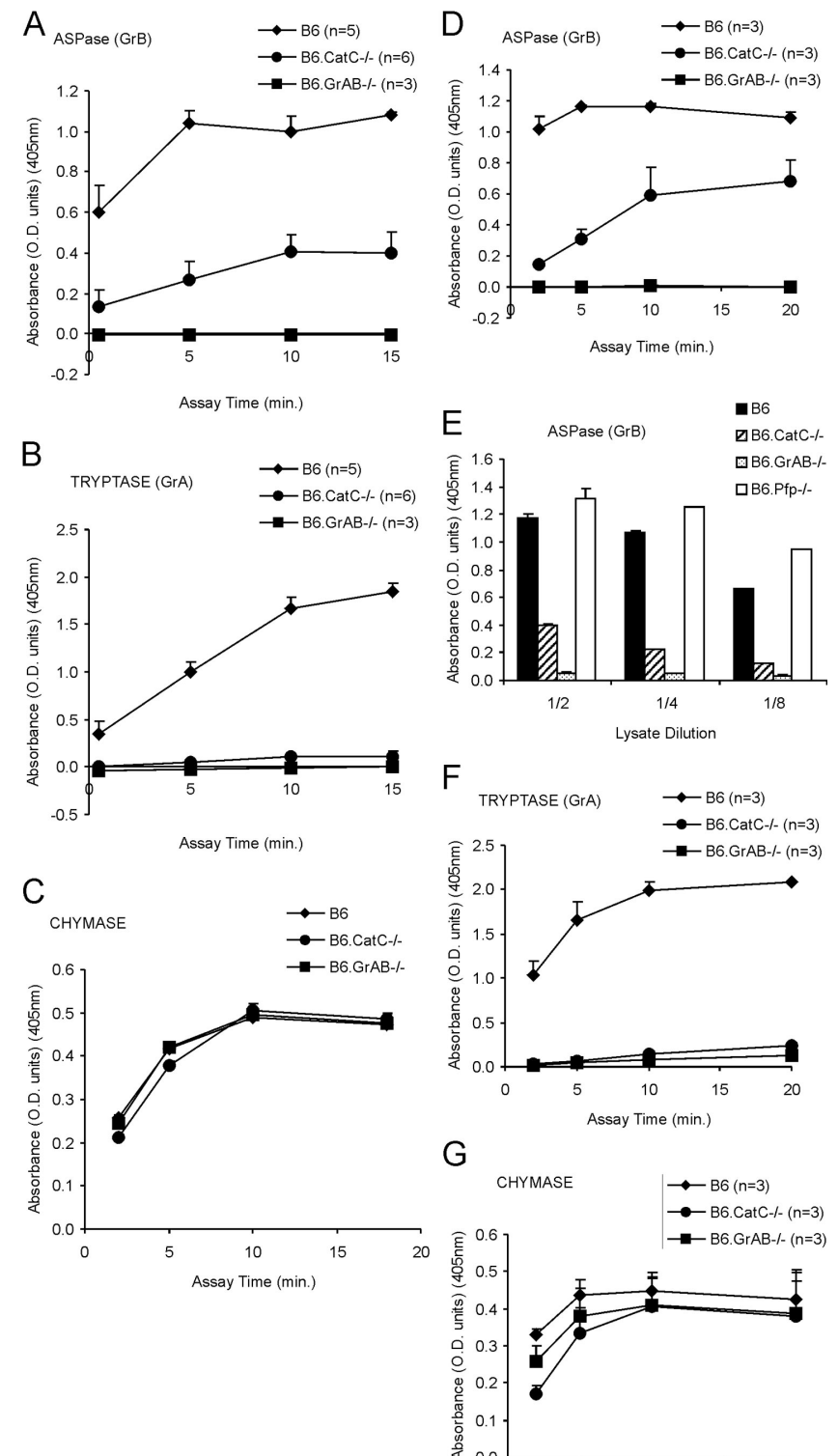

$\mathrm{F}$

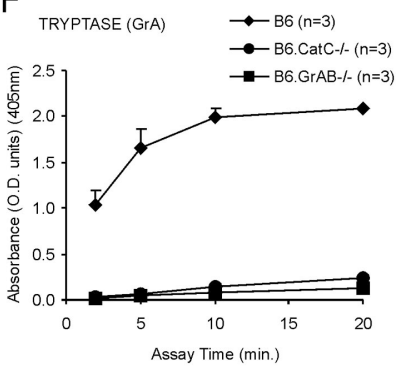

G

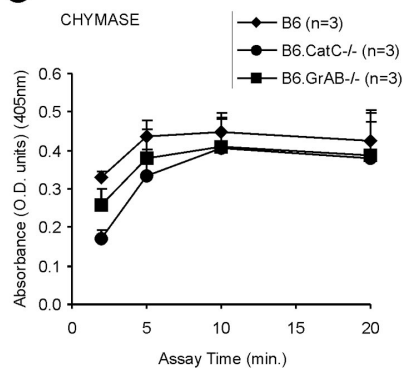

Figure 3. Serine protease activities in CatC-null and -expressing day 7 primary and day 3 secondary allo-stimulated $\mathrm{CD}^{+}$lymphocytes. Approximately $20 \mu \mathrm{g}$ of whole cell lysate protein prepared from purified CD8 ${ }^{+}$ $T$ cells from day 7 primary (A-C) or day 3 secondary (D-G) CTLs were assayed for various granzyme activities as a function of time. In $A, D$, and $E$, ASPase activity was measured by hydrolysis of the peptide thioester substrate Boc-Ala-Ala-Asp-S-Bzl, which is specifically cleaved by Gr B. In E, single lysates from one of the experiments shown in $D$ were progressively diluted and tested in triplicate for $15 \mathrm{~min}$. (B and F) Tryptase activity was measured by cleavage of BLT; (C and G) chymotrypsin-like (chymase) activity was measured by cleavage of Suc-Phe-Leu-Phe-SBzl. The data points show the mean of independent experiments (each of which was performed in triplicate) \pm SEM. The number of independent experiments performed for each effector type is indicated in the figure.

primary infection by the natural mouse pathogen ECTV, an orthopoxvirus (Mullbacher et al., 1999a,b). These mice are unable to control ECTV replication, and viral load in their spleen is several orders of magnitude higher than B6 mice (Mullbacher, 2003). We therefore estimated viral titers in the spleens and livers of CatC-null and -sufficient B6 mice after
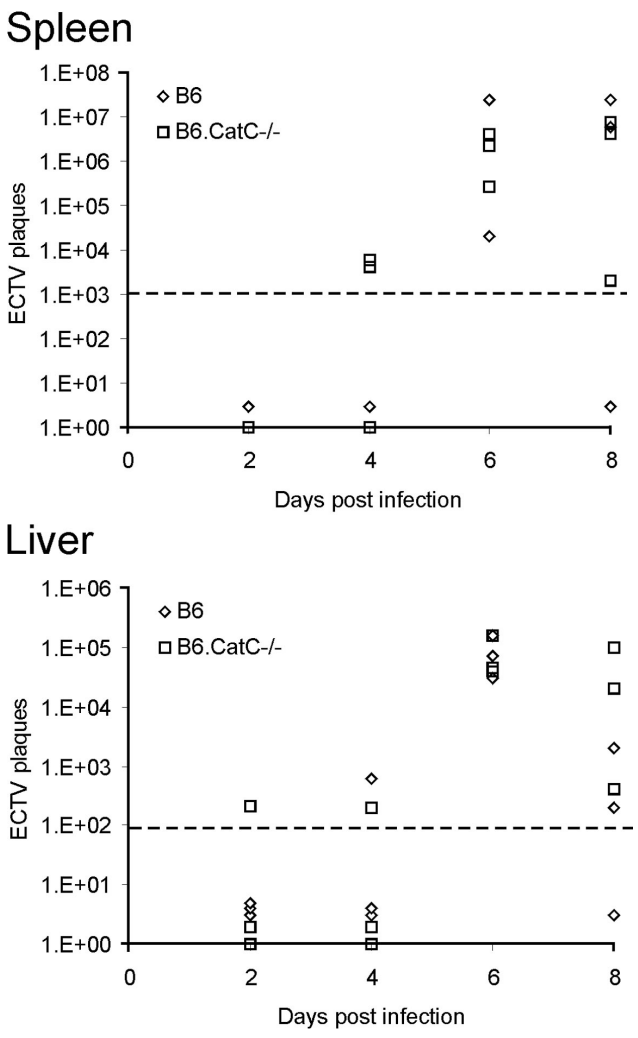

Figure 4. ECTV titers in the spleens and livers of CatC-expressing and -null mice. Mice were infected in the hind footpads with ECTV as described in Materials and methods. Groups of three mice were killed on days 2, 4, 6, and 8 after infection, and their organs were assayed for virus titers by plaque assay on BSC-1 cell monolayers. In each panel, the horizontal line indicates the detection limit of the assay.

ECTV infection into the footpad (Fig. 4). Viral titers in spleen and liver were estimated at 2, 4, 6, and $8 \mathrm{~d}$ after infection and showed no measurable difference at any time point. In contrast, deficiency of both GrA and -B has been shown to increase viral titer in the liver and spleen $\sim 10,000$-fold by days 6 and 8 , compared with granzyme-expressing mice (Mullbacher et al., 1999b). In addition, we found that the CatC-null mice had only a slightly higher mortality than wild-type mice, but far lower than that seen in perforin or GrAB-deficient mice (unpublished data). Overall, our data indicated that the phenotype of CatCnull mice with respect to ECTV infection in vivo is distinct from that of perforin or GrAB-deficient mice on the same genetic background; namely, perforin- and GrAB gene-disrupted mice are far more susceptible.

\section{Discussion}

The lymphocyte and myeloid cell serine proteases are unusual amongst proteolytic enzymes in that they are stored in secretory lysosomes as active enzymes (Salvesen and Enghild, 1990). Activation is a two-step process and involves removal of the leader sequence followed by cleavage of two final residues at the $\mathrm{N}$ terminus at the time of granule packaging (Masson and Tschopp, 1987; Bleackley et al., 1988; Caputo et al., 1993). Studies using protease inhibitors or in which purified CatC 
was added to recombinant granzymes (Caputo et al., 1993; McGuire et al., 1993; Smyth et al., 1995; Kummer et al., 1996; Pham et al., 1998) or, finally, with CatC-knockout mice (Pham and Ley, 1999) indicated that CatC is the principal protease that removes the activation dipeptide. Our current study clearly confirms that CatC is responsible for virtually all of the GrA and most of the GrB activity in CTLs. However, our results also clearly indicate that not all of the GrB activity is eliminated in $\mathrm{CatC}^{-1-}$ mice and that the extent of the immune defect of CatCnull mice may have been overestimated in the past.

Surprisingly, we have found that although CatC seems to be critical for activating GrA, it is not essential for GrB activity. As a result, CatC-deficient mice retain substantial CTL cytotoxicity, inducing apoptosis in vivo and in vitro. The reduced GrB activity of $\mathrm{CatC}^{-1-}$ CTLs was still sufficient to induce DNA fragmentation in target cells that was comparable to that induced by wild-type effector cells. This indicated that the quantity of GrB released by wild-type CTLs is well in excess of the amount required to trigger DNA fragmentation in P815 cells. Much of the DNA fragmentation induced by GrB is normally reliant on caspase activation (Sarin et al., 1998; Trapani et al., 1998a), so the sufficiency of $\mathrm{CatC}^{-/-}$CTLs in inducing DNA fragmentation indicates that the quantity of GrB delivered by these cells is adequate to effectively initiate caspase processing. Similarly, live-cell imaging demonstrated that target cells exposed to $\mathrm{CatC}^{-/-}$CTLs died by classic apoptosis: both the morphology and the acquisition of apoptotic markers (phosphatidylserine exposure, PI uptake) as a function of time were indistinguishable between cells incubated with CatC-deficient or -sufficient killer cells. In vivo, CatC-null mice were far less susceptible to both $\mathrm{GrAB}^{-1-}$ and $\mathrm{pfp}^{-1-}$ mice to ECTV, and viral titers generated in their livers and spleens were comparable to those in wild-type B6 mice. Indeed, the kinetics of virus replication in $\mathrm{CatC}^{-1-}$ mice matched that seen with $\mathrm{GrA}^{-/-}$mice (Mullbacher et al., 1996,b), which is consistent with our findings that the $\mathrm{CatC}^{-/-}$lacked GrA expression but retained measurable GrB activity. Overall, the current study makes it clear that CatC-null mice are considerably less immunologically compromised than either GrA/B-cluster or perforin-deficient mice and that alternative mechanisms exist for GrB activation in the absence of CatC.

A lack of neutrophil serine protease activity has been observed in $\mathrm{CatC}^{-/-}$mice (Adkison et al., 2002), as was confirmed in our study. A role for these proteases in defense against bacterial and fungal pathogens has been suggested (Belaaouaj et al., 1998; Tkalcevic et al., 2000), but the only study that addressed this issue, surprisingly, showed that the absence of CatC contributed to increased survival from sepsis (Mallen-St Clair et al., 2004). A deficiency of cell-mediated immunity is not a prominent feature of CatC deficiency in humans. Most CatC-deficient patients present with Papillon-Lefevre syndrome (PLS), a rare autosomal recessive disorder (Gorlin et al., 1964; Hart et al., 1999) marked by severe, early onset periodontal disease, with subsequent premature loss of both primary and secondary dentition, and palmoplantar hyperkeratosis (Nuckolls and Slavkin, 1999; Toomes et al., 1999). Although the critical CatC substrates responsible for PLS have not been defined, it has been suggested that CatC may play a role in maintaining the structure and integrity of the epidermis surrounding the teeth (Nuckolls and Slavkin, 1999; Lundgren et al., 2005). An increased susceptibility to bacterial infections both in the mouth and within organs such as the liver has been reported and may reflect defects in neutrophil activity, manifested as an absence of CatG, NE, and protease 3 activity (de Haar et al., 2004; Pham et al., 2004; Lundgren et al., 2005). Recent reports have produced somewhat conflicting results with regard to lymphocyte function in PLS patients. Three of the seven patients described in the aforementioned studies also had a slight reduction in GrA protein levels, but considerable GrA and -B activities were detected (Pham et al., 2004), and IL-2-activated T cells generated from these patients were able to efficiently kill K562 target cells. However, naive NK cells isolated from a further 20 PLS patients (representing seven families) displayed an $\sim 50 \%$ reduction in activity against K562, compared with a pool of normal healthy controls (Lundgren et al., 2005). The profile of effector molecules expressed by these two cell types is distinct, perhaps accounting for the apparently discordant results (Sedelies et al., 2004). In particular, resting NK cells have very low levels of GrB but a very high constitutive level of $\mathrm{GrH}$, whereas $\mathrm{GrB}$ expression increases markedly with IL-2 stimulation (Sedelies et al., 2004). In another study, it was shown that the NK cells of a PLS patient exhibited GrB activity after stimulation with IL-2 (Meade et al., 2006). We have found similar results in CatC-null NK cells (unpublished data).

CatC, an amino-dipeptidase, belongs to a family of lysosomal papain-like cysteine proteases (Rao et al., 1997). Unlike related proteases, which function as monomeric endopeptidases, CatC is an oligomeric exopeptidase with a general preference for acidic N-terminal sequences (see below) until it encounters a "stop sequence," usually a charged residue, such as arginine, lysine, or proline (Tran et al., 2002). However, CatC is also well recognized for its ability to cleave the dipeptide at the $\mathrm{N}$ terminus of serine proteases found in lymphocytes and neutrophils. Exposure of the invariant $\mathrm{N}$-terminal isoleucine in all granzymes allows it to form an ion pair with the aspartate within the catalytic pocket, which leads to the formation of a functional catalytic center (Salvesen and Enghild, 1990). It is unclear whether further trimming is prevented, as the isoleucine is seen as a stop sequence, or whether the ion pair formation is instantaneous, which makes the $\mathrm{N}$ terminus unavailable for further processing. In the absence of $\mathrm{CatC}$, other less specific lysosomal enzymes could potentially remove the activation dipeptide. However, this degree of redundancy is limited, as we showed that GrB activity remains markedly compromised, whereas GrA activity remains almost completely absent. Interestingly, an analogous study by Wolters et al. (2001) showed that tryptases but not chymases are still activated in $\mathrm{CatC}^{-1-}$ mast cells. Although Pham and Ley (1999) were unable to find evidence for dipeptide-deleted GrA and -B, they did, surprisingly, find that $50 \%$ of the $\mathrm{GrC}$ they were able to purify from CatC-deficient CTLs had had its activation dipeptide removed, and they suggested that an alternative protease, DPPIV, might be responsible. This seems unlikely to us, as substrate analysis has suggested that DPPIV has a strict preference for proline in 
the $\mathrm{P} 1$ position, although alanine was tolerated in two identified substrates (Leiting et al., 2003). Comparison of the dipeptide sequence in serine proteases found that all of the granzymes, with the exception of GrA, have an acidic residue (Glu) in the P1 position (Lobe et al., 1986; Jenne et al., 1988a,b; Jenne et al., 1989), as do the mast cell chymases (McGuire et al., 1993; Dikov et al., 1994), carboxypeptidase A (Reynolds et al., 1989), and NE (Sturrock et al., 1998). In contrast, GrA has arginine (Hershberger et al., 1992), CatG has lysine (Heusel et al., 1993), and the mast cell tryptases have glycine (Dikov et al., 1994). Therefore, in terms of the specificity of an alternative dipeptidase acting in the absence of $\mathrm{CatC}$, there is not a consistent preference for a specific residue in the $\mathrm{P} 1$ position. We are currently attempting to identify the proteases responsible for partially compensating for CatC's absence, using biochemical and genetic approaches.

\section{Materials and methods}

\section{Mice}

Inbred $\mathrm{B} 6\left(\mathrm{H}-2^{b}\right), \mathrm{BALB} / \mathrm{c}\left(\mathrm{H}-2^{\mathrm{d}}\right)$, and $\mathrm{C} 3 \mathrm{H} / \mathrm{J}\left(\mathrm{H}-2^{k}\right)$ mice were purchased from the Walter and Eliza Hall Institute of Medical Research. GrA and GrB cluster-deficient $\left(\mathrm{GrAB}^{-/-}\right)$mice (Simon et al., 1997) were obtained from M. Simon (Max-Planck-Institut für Immunbiologie, Freiburg, Germany) and were maintained at the Peter MacCallum Cancer Centre. Mice were genotyped using the PCR screening protocols previously described (Simon et al., 1997). Originally, the $\mathrm{GrA}^{-/-}$mice (Ebnet et al., 1995) were derived on the B6 background and the GrB cluster-deficient mice (Heusel et al., 1994) on the 129 background. After appropriate matings and selection of $\mathrm{GrAB}^{-1-}$ progeny, the mice were backcrossed for eight generations to $\mathrm{B} 6$. The CatC (dipeptidyl peptidase 1) knockout (B6.CatC ${ }^{-1-}$ ) mice (Pham and Ley, 1999) were provided by C. Pham (Washington University School of Medicine, St. Louis, MO) and maintained at the Peter MacCallum Cancer Centre. The B6. $\mathrm{CatC}^{-1-}$ mice had previously been backcrossed to $\mathrm{B} 6$ for 11 generations. We used a PCR screening protocol provided by C. Pham to confirm disruption of the CatC gene and acquisition of the Lac $Z$ cassette in knockout animals (Pham and Ley, 1999). We also recapitulated the myeloid phenotype of the mice by confirming the absence of active CatG and NE from neutrophils (Fig. S2; see the following paragraph). Mice 5-10 wk of age were used in all experiments, and the studies conformed to Peter MacCallum Cancer Centre and the John Curtin School of Medical Research animal experimental ethics committee guidelines.

\section{Enzyme activity assays}

Whole cell lysates of CTLs generated in mixed lymphocyte cultures were normalized for protein content and analyzed for granule serine protease activity by the hydrolysis of synthetic peptide thiobenzylester substrates: for ASPase activity, Boc-Ala-Ala-Asp-S-Bzl (a gift from J. Powers, Georgia Institute of Technology, Atlanta, GA); for tryptase activity, BLT (Sigma-Aldrich); and for chymotrypsin-like (chymase) activity, Suc-Phe-Leu-Phe-SBzl (Enzyme Systems Products), as described previously (Edwards et al., 1999; Davis et al., 2003). The failure of $\mathrm{CatC}^{-1-}$ mice to activate the bone marrowderived serine proteases NE and CatG was confirmed by the lack of cleavage of specific peptide paranitroanilide substrates in chromogenic assays as previously described (Maclvor et al., 1999; Adkison et al., 2002). Absorbance readings (at $405 \mathrm{nM}$ ) were taken at $15 \mathrm{~min}$. The data points shown represent the means \pm SEM.

\section{Cell culture and reagents}

P815 (mouse mastocytoma; $\mathrm{H}-2^{d}$ ) and MS9II (mouse fibroblast; $\mathrm{H}-2^{k}$ ) cells were maintained in DME (JRH Bioscience) supplemented with 10\% (vol/ vol) FCS (JRH Bioscience), $2 \mathrm{mM}$ glutamine (JRH Bioscience), $100 \mathrm{U} / \mathrm{ml}$ penicillin, $100 \mu \mathrm{g} / \mathrm{ml}$ streptomycin (Invitrogen), and $1 \mathrm{mM}$ sodium pyruvate (Invitrogen). Primary mouse CTLs were maintained in supplemented RPMI (Invitrogen) also containing $100 \mu \mathrm{M}$ nonessential amino acids and $0.1 \mathrm{mM}$ 2-mercapto-ethanol. Western blot analysis (Sutton et al., 2000) was used to confirm perforin expression using the rat PI-8 antibody (Kamiya Biomedical; Kawasaki et al., 1990) and GrB expression using the rat anti-GrB antibody (eBioscience).

\section{Generation of cytotoxic lymphocytes}

Allogenic CTLs capable of recognizing $\mathrm{H}-2^{\mathrm{d}}$ expressed on P815 cells or $\mathrm{H}-2^{\mathrm{k}}$ on MS9ll cells were generated in one way in vitro mixed lymphocyte reactions. Splenocytes isolated from B6 and the gene-targeted mouse strains listed above were cultured for $7 \mathrm{~d}$ in RPMI medium with lethally irradiated $\mathrm{BALB} / \mathrm{c}\left(\mathrm{H}-2^{\mathrm{d}}\right)$ or $\mathrm{C} 3 \mathrm{H} / \mathrm{J}\left(\mathrm{H}-2^{k}\right)$ splenocytes. The stimulator/responder cell ratio was $1: 1$, and responder cells were seeded at 2 eb cells per milliliter of culture medium in 24-well plates. In some experiments, the cells were restimulated in a similar fashion for a further $3 \mathrm{~d}$. Cell death induced by CTL populations was assessed by ${ }^{51} \mathrm{Cr}$ and ${ }^{125}$ I-DNA release, as described previously (Sutton et al., 1997).

\section{Time-lapse confocal microscopy}

Time-lapse confocal microscopy was performed essentially as previously described (Waterhouse et al., 2006). In brief, MS9II cells were plated and allowed to adhere to 96-well culture plates and incubated overnight at $37^{\circ} \mathrm{C}$ in a humidified $\mathrm{CO}_{2}$ incubator. The plates were transferred to a temperature-controlled stage (Prior Proscan) maintained at $37^{\circ} \mathrm{C}$ on a microscope (IX-81; Olympus). PI (Sigma-Aldrich) was added to the cultures, in tissue culture medium (DME) at $50 \mathrm{ng} / \mathrm{ml}$ and AV-FLUOS (Roche) at $2 \mu \mathrm{g} / \mathrm{ml}$. Cells were exposed to an equal number of activated CD8 ${ }^{+}$CTLs and viewed for the times indicated. Images were captured at specified intervals using a charge-coupled device camera (ORCA-ER; Hamamatsu) controlled by MetaMorph software (Universal Imaging Corp.). The images were viewed with an LCPlanFl objective lens at $20 \times(\mathrm{NA} 0.4)$. As the fluorescence intensities of AV-FLUOS and PI varied, we plotted the fluorescence reading for each frame relative to the maximum for that fluorochrome over the entire time course (using MetaMorph and Excel [Microsoft]), after subtraction of background fluorescence at each time point. For each fluorophore, the maximum fluorescence plotted was therefore defined as 1.0, and baseline fluorescence was 0 .

\section{Infection with ECTV in vivo}

The kinetics of ECTV replication were estimated as previously described (Mullbacher et al., 1996). Mice were infected with $10^{3}$ pfu ECTV (Moscow strain) into the footpad. Three mice were killed on days 2, 4, 6, and 8 after infection, and liver, spleen, and blood were harvested. The viral titers in spleen and liver were determined as described previously (Mullbacher et al., 1996).

\section{Online supplemental material}

Fig. S1 demonstrates a chromium release assay showing that apoptosis induced by $C D 8^{+}$CTLs is granule mediated. Fig. S2 demonstrates a chromogenic substrate cleavage assay showing CatG and NE activities in CatC-null and -expressing neutrophils. Video 1 is a time-lapse video of CatC-null CTLinduced death of an MS9II target cell. Online supplemental material is available at http://www.jcb.org/cgi/content/full/jcb.200609077/DC1.

We thank Dr. Christine Pham for the generous gift of CatC-deficient mice and Ms. Jean Hendy for technical assistance.

J.A. Trapani and V.R. Sutton are supported by a program grant from the National Health and Medical Research Council (NHMRC; 454569) of Australia. J.A. Trapani received a senior fellowship (288999) and N.J. Waterhouse a career development award (165405) from NHMRC.

Submitted: 13 September 2006

Accepted: 6 January 2007

\section{References}

Adkison, A.M., S.Z. Raptis, D.G. Kelley, and C.T. Pham. 2002. Dipeptidyl peptidase I activates neutrophil-derived serine proteases and regulates the development of acute experimental arthritis. J. Clin. Invest. 109:363-371.

Belaaouaj, A., R. McCarthy, M. Baumann, Z. Gao, T.J. Ley, S.N. Abraham, and S.D. Shapiro. 1998. Mice lacking neutrophil elastase reveal impaired host defense against gram negative bacterial sepsis. Nat. Med. 4:615-618.

Beresford, P.J., Z. Xia, A.H. Greenberg, and J. Lieberman. 1999. Granzyme A loading induces rapid cytolysis and a novel form of DNA damage independently of caspase activation. Immunity. 10:585-594.

Bleackley, R.C., B. Duggan, N. Ehrman, and C.G. Lobe. 1988. Isolation of two cDNA sequences which encode cytotoxic cell proteases. FEBS Lett. 234:153-159.

Browne, K.A., E. Blink, V.R. Sutton, C.J. Froelich, D.A. Jans, and J.A. Trapani. 1999. Cytosolic delivery of granzyme B by bacterial toxins: evidence that 
endosomal disruption, in addition to transmembrane pore formation, is an important function of perforin. Mol. Cell. Biol. 19:8604-8615.

Caputo, A., R.S. Garner, U. Winkler, D. Hudig, and R.C. Bleackley. 1993. Activation of recombinant murine cytotoxic cell proteinase-1 requires deletion of an amino-terminal dipeptide. J. Biol. Chem. 268:17672-17675.

Davis, J.E., V.R. Sutton, K.A. Browne, and J.A. Trapani. 2003. Purification of natural killer cell cytotoxic granules for assaying target cell apoptosis. J. Immunol. Methods. 276:59-68.

de Haar, S.F., D.C. Jansen, T. Schoenmaker, H. De Vree, V. Everts, and W. Beertsen. 2004. Loss-of-function mutations in cathepsin $\mathrm{C}$ in two families with Papillon-Lefevre syndrome are associated with deficiency of serine proteinases in PMNs. Hum. Mutat. 23:524.

Dikov, M.M., E.B. Springman, S. Yeola, and W.E. Serafin. 1994. Processing of procarboxypeptidase A and other zymogens in murine mast cells. J. Biol. Chem. 269:25897-25904.

Duke, R.C., P.M. Persechini, S. Chang, C.C. Liu, J.J. Cohen, and J.D. Young. 1989. Purified perforin induces target cell lysis but not DNA fragmentation. J. Exp. Med. 170:1451-1456.

Ebnet, K., M. Hausmann, F. Lehmann-Grube, A. Mullbacher, M. Kopf, M. Lamers, and M.M. Simon. 1995. Granzyme A-deficient mice retain potent cell-mediated cytotoxicity. EMBO J. 14:4230-4239.

Edwards, K.M., C.M. Kam, J.C. Powers, and J.A. Trapani. 1999. The human cytotoxic $\mathrm{T}$ cell granule serine protease granzyme $\mathrm{H}$ has chymotrypsin-like (chymase) activity and is taken up into cytoplasmic vesicles reminiscent of granzyme B-containing endosomes. J. Biol. Chem. 274:30468-30473.

Froelich, C.J., K. Orth, J. Turbov, P. Seth, R. Gottlieb, B. Babior, G.M. Shah, R.C. Bleackley, V.M. Dixit, and W. Hanna. 1996. New paradigm for lymphocyte granule-mediated cytotoxicity. Target cells bind and internalize granzyme B, but an endosomolytic agent is necessary for cytosolic delivery and subsequent apoptosis. J. Biol. Chem. 271:29073-29079.

Gorlin, R.J., H. Sedano, and V.E. Anderson. 1964. The syndrome of PalmarPlantar hyperkeratosis and premature periodontal destruction of the teeth. A clinical and genetic analysis of the Papillon-Lef'evre syndrome. J. Pediatr. 65:895-908.

Hart, T.C., P.S. Hart, D.W. Bowden, M.D. Michalec, S.A. Callison, S.J. Walker, Y. Zhang, and E. Firatli. 1999. Mutations of the cathepsin C gene are responsible for Papillon-Lefevre syndrome. J. Med. Genet. 36:881-887.

Hershberger, R.J., H.K. Gershenfeld, I.L. Weissman, and L. Su. 1992. Genomic organization of the mouse granzyme A gene. Two mRNAs encode the same mature granzyme A with different leader peptides. J. Biol. Chem. 267:25488-25493.

Heusel, J.W., E.M. Scarpati, N.A. Jenkins, D.J. Gilbert, N.G. Copeland, S.D. Shapiro, and T.J. Ley. 1993. Molecular cloning, chromosomal location, and tissue-specific expression of the murine cathepsin G gene. Blood. 81:1614-1623.

Heusel, J.W., R.L. Wesselschmidt, S. Shresta, J.H. Russell, and T.J. Ley. 1994. Cytotoxic lymphocytes require granzyme B for the rapid induction of DNA fragmentation and apoptosis in allogeneic target cells. Cell. 76:977-987.

Jenne, D., C. Rey, J.A. Haefliger, B.Y. Qiao, P. Groscurth, and J. Tschopp. 1988a. Identification and sequencing of cDNA clones encoding the granule-associated serine proteases granzymes $\mathrm{D}, \mathrm{E}$, and $\mathrm{F}$ of cytolytic T lymphocytes. Proc. Natl. Acad. Sci. USA. 85:4814-4818.

Jenne, D., C. Rey, D. Masson, K.K. Stanley, J. Herz, G. Plaetinck, and J. Tschopp. 1988b. cDNA cloning of granzyme C, a granule-associated serine protease of cytolytic T lymphocytes. J. Immunol. 140:318-323.

Jenne, D.E., D. Masson, M. Zimmer, J.A. Haefliger, W.H. Li, and J. Tschopp. 1989. Isolation and complete structure of the lymphocyte serine protease granzyme $\mathrm{G}$, a novel member of the granzyme multigene family in murine cytolytic T lymphocytes. Evolutionary origin of lymphocyte proteases. Biochemistry. 28:7953-7961.

Johnson, H., L. Scorrano, S.J. Korsmeyer, and T.J. Ley. 2003. Cell death induced by granzyme C. Blood. 101:3093-3101.

Kagi, D., B. Ledermann, K. Burki, P. Seiler, B. Odermatt, K.J. Olsen, E.R. Podack, R.M. Zinkernagel, and H. Hengartner. 1994. Cytotoxicity mediated by $\mathrm{T}$ cells and natural killer cells is greatly impaired in perforindeficient mice. Nature. 369:31-37.

Kawasaki, A., Y. Shinkai, Y. Kuwana, A. Furuya, Y. Iigo, N. Hanai, S. Itoh, H. Yagita, and K. Okumura. 1990. Perforin, a pore-forming protein detectable by monoclonal antibodies, is a functional marker for killer cells. Int. Immunol. 2:677-684.

Kelly, J.M., N.J. Waterhouse, E. Cretney, K.A. Browne, S. Ellis, J.A. Trapani, and M.J. Smyth. 2004. Granzyme M mediates a novel form of perforindependent cell death. J. Biol. Chem. 279:22236-22242.

Kummer, J.A., A.M. Kamp, F. Citarella, A.J. Horrevoets, and C.E. Hack. 1996. Expression of human recombinant granzyme A zymogen and its activation by the cysteine proteinase cathepsin C. J. Biol. Chem. 271:9281-9286.
Leiting, B., K.D. Pryor, J.K. Wu, F. Marsilio, R.A. Patel, C.S. Craik, J.A. Ellman, R.T. Cummings, and N.A. Thornberry. 2003. Catalytic properties and inhibition of proline-specific dipeptidyl peptidases II, IV and VII. Biochem. J. 371:525-532.

Lieberman, J., and Z. Fan. 2003. Nuclear war: the granzyme A-bomb. Curr. Opin. Immunol. 15:553-559.

Lobe, C.G., B.B. Finlay, W. Paranchych, V.H. Paetkau, and R.C. Bleackley. 1986. Novel serine proteases encoded by two cytotoxic T lymphocytespecific genes. Science. 232:858-861.

Lu, H., Q. Hou, T. Zhao, H. Zhang, Q. Zhang, L. Wu, and Z. Fan. 2006. Granzyme $M$ directly cleaves inhibitor of caspase-activated DNase (CAD) to unleash CAD leading to DNA fragmentation. J. Immunol. 177:1171-1178.

Lundgren, T., R.S. Parhar, S. Renvert, and D.N. Tatakis. 2005. Impaired cytotoxicity in Papillon-Lefevre syndrome. J. Dent. Res. 84:414-417.

MacIvor, D.M., S.D. Shapiro, C.T. Pham, A. Belaaouaj, S.N. Abraham, and T.J. Ley. 1999. Normal neutrophil function in cathepsin G-deficient mice. Blood. 94:4282-4293.

Mallen-St Clair, J., C.T. Pham, S.A. Villalta, G.H. Caughey, and P.J. Wolters. 2004. Mast cell dipeptidyl peptidase I mediates survival from sepsis. J. Clin. Invest. 113:628-634.

Masson, D., and J. Tschopp. 1987. A family of serine esterases in lytic granules of cytolytic T lymphocytes. Cell. 49:679-685.

McGuire, M.J., P.E. Lipsky, and D.L. Thiele. 1993. Generation of active myeloid and lymphoid granule serine proteases requires processing by the granule thiol protease dipeptidyl peptidase I. J. Biol. Chem. 268:2458-2467.

Meade, J.L., E.A. de Wynter, P. Brett, S. Malik Sharif, C.G. Woods, A.F. Markham, and G.P. Cook. 2006. A family with Papillon-Lefevre syndrome reveals a requirement for cathepsin $\mathrm{C}$ in granzyme $\mathrm{B}$ activation and NK cell cytolytic activity. Blood. 107:3665-3668.

Mullbacher, A. 2003. Cell-mediated cytotoxicity in recovery from poxvirus infections. Rev. Med. Virol. 13:223-232.

Mullbacher, A., K. Ebnet, R.V. Blanden, R.T. Hla, T. Stehle, C. Museteanu, and M.M. Simon. 1996. Granzyme A is critical for recovery of mice from infection with the natural cytopathic viral pathogen, ectromelia. Proc. Natl. Acad. Sci. USA. 93:5783-5787.

Mullbacher, A., R.T. Hla, C. Museteanu, and M.M. Simon. 1999a. Perforin is essential for control of ectromelia virus but not related poxviruses in mice. J. Virol. 73:1665-1667.

Mullbacher, A., P. Waring, R. Tha Hla, T. Tran, S. Chin, T. Stehle, C. Museteanu, and M.M. Simon. 1999b. Granzymes are the essential downstream effector molecules for the control of primary virus infections by cytolytic leukocytes. Proc. Natl. Acad. Sci. USA. 96:13950-13955.

Nuckolls, G.H., and H.C. Slavkin. 1999. Paths of glorious proteases. Nat. Genet. 23:378-380.

Pardo, J., S. Balkow, A. Anel, and M.M. Simon. 2002. The differential contribution of granzyme A and granzyme B in cytotoxic T lymphocyte-mediated apoptosis is determined by the quality of target cells. Eur. J. Immunol. 32:1980-1985.

Pham, C.T., and T.J. Ley. 1999. Dipeptidyl peptidase I is required for the processing and activation of granzymes A and B in vivo. Proc. Natl. Acad. Sci. USA. 96:8627-8632.

Pham, C.T., D.A. Thomas, J.D. Mercer, and T.J. Ley. 1998. Production of fully active recombinant murine granzyme B in yeast. J. Biol. Chem. 273:1629-1633.

Pham, C.T., J.L. Ivanovich, S.Z. Raptis, B. Zehnbauer, and T.J. Ley. 2004. Papillon-Lefevre syndrome: correlating the molecular, cellular, and clinical consequences of cathepsin C/dipeptidyl peptidase I deficiency in humans. J. Immunol. 173:7277-7281.

Podack, E.R. 1999. How to induce involuntary suicide: the need for dipeptidyl peptidase I. Proc. Natl. Acad. Sci. USA. 96:8312-8314.

Rao, N.V., G.V. Rao, and J.R. Hoidal. 1997. Human dipeptidyl-peptidase I. Gene characterization, localization, and expression. J. Biol. Chem. 272:10260-10265.

Reynolds, D.S., D.S. Gurley, R.L. Stevens, D.J. Sugarbaker, K.F. Austen, and W.E. Serafin. 1989. Cloning of cDNAs that encode human mast cell carboxypeptidase $\mathrm{A}$, and comparison of the protein with mouse mast cell carboxypeptidase A and rat pancreatic carboxypeptidases. Proc. Natl. Acad. Sci. USA. 86:9480-9484.

Salvesen, G., and J.J. Enghild. 1990. An unusual specificity in the activation of neutrophil serine proteinase zymogens. Biochemistry. 29:5304-5308.

Sarin, A., E.K. Haddad, and P.A. Henkart. 1998. Caspase dependence of target cell damage induced by cytotoxic lymphocytes. J. Immunol. 161:2810-2816.

Sedelies, K.A., T.J. Sayers, K.M. Edwards, W. Chen, D.G. Pellicci, D.I. Godfrey, and J.A. Trapani. 2004. Discordant regulation of granzyme $\mathrm{H}$ and granzyme B expression in human lymphocytes. J. Biol. Chem. 279:26581-26587. 
Shi, L., R.P. Kraut, R. Aebersold, and A.H. Greenberg. 1992. A natural killer cell granule protein that induces DNA fragmentation and apoptosis. J. Exp. Med. 175:553-566.

Simon, M.M., M. Hausmann, T. Tran, K. Ebnet, J. Tschopp, R. ThaHla, and A. Mullbacher. 1997. In vitro- and ex vivo-derived cytolytic leukocytes from granzyme $\mathrm{A} \times \mathrm{B}$ double knockout mice are defective in granule-mediated apoptosis but not lysis of target cells. J. Exp. Med. 186:1781-1786.

Smyth, M.J., M.J. McGuire, and K.Y. Thia. 1995. Expression of recombinant human granzyme B. A processing and activation role for dipeptidyl peptidase I. J. Immunol. 154:6299-6305.

Stepp, S.E., R. Dufourcq-Lagelouse, F. Le Deist, S. Bhawan, S. Certain, P.A Mathew, J.I. Henter, M. Bennett, A. Fischer, G. de Saint Basile, and V. Kumar. 1999. Perforin gene defects in familial hemophagocytic lymphohistiocytosis. Science. 286:1957-1959.

Sturrock, A., K.F. Franklin, S. Wu, and J.R. Hoidal. 1998. Characterization and localization of the genes for mouse proteinase-3 (Prtn3) and neutrophil elastase (Ela2). Cytogenet. Cell Genet. 83:104-108.

Sutton, V.R., D.L. Vaux, and J.A. Trapani. 1997. Bcl-2 prevents apoptosis induced by perforin and granzyme B, but not that mediated by whole cytotoxic lymphocytes. J. Immunol. 158:5783-5790.

Sutton, V.R., J.E. Davis, M. Cancilla, R.W. Johnstone, A.A. Ruefli, K. Sedelies, K.A. Browne, and J.A. Trapani. 2000. Initiation of apoptosis by granzyme B requires direct cleavage of bid, but not direct granzyme B-mediated caspase activation. J. Exp. Med. 192:1403-1414.

Tkalcevic, J., M. Novelli, M. Phylactides, J.P. Iredale, A.W. Segal, and J. Roes 2000. Impaired immunity and enhanced resistance to endotoxin in the absence of neutrophil elastase and cathepsin G. Immunity. 12:201-210.

Toomes, C., J. James, A.J. Wood, C.L. Wu, D. McCormick, N. Lench, C. Hewitt, L. Moynihan, E. Roberts, C.G. Woods, et al. 1999. Loss-of-function mutations in the cathepsin $\mathrm{C}$ gene result in periodontal disease and palmoplantar keratosis. Nat. Genet. 23:421-424.

Tran, T.V., K.A. Ellis, C.M. Kam, D. Hudig, and J.C. Powers. 2002. Dipeptidyl peptidase I: importance of progranzyme activation sequences, other dipeptide sequences, and the N-terminal amino group of synthetic substrates for enzyme activity. Arch. Biochem. Biophys. 403:160-170.

Trapani, J.A. 1998. Dual mechanisms of apoptosis induction by cytotoxic lymphocytes. Int. Rev. Cytol. 182:111-192.

Trapani, J.A., and M.J. Smyth. 2002. Functional significance of the perforin/ granzyme cell death pathway. Nat. Rev. Immunol. 2:735-747.

Trapani, J.A., D.A. Jans, P.J. Jans, M.J. Smyth, K.A. Browne, and V.R. Sutton 1998a. Efficient nuclear targeting of granzyme B and the nuclear consequences of apoptosis induced by granzyme B and perforin are caspase-dependent, but cell death is caspase-independent. J. Biol. Chem. 273:27934-27938.

Trapani, J.A., P. Jans, M.J. Smyth, C.J. Froelich, E.A. Williams, V.R. Sutton, and D.A. Jans. 1998b. Perforin-dependent nuclear entry of granzyme $\mathrm{B}$ precedes apoptosis, and is not a consequence of nuclear membrane dysfunction. Cell Death Differ. 5:488-496.

Voskoboinik, I., M.C. Thia, A. De Bono, K. Browne, E. Cretney, J.T. Jackson, P.K. Darcy, S.M. Jane, M.J. Smyth, and J.A. Trapani. 2004. The functional basis for hemophagocytic lymphohistiocytosis in a patient with co-inherited missense mutations in the perforin (PFN1) gene. J. Exp. Med. 200:811-816.

Waterhouse, N.J., V.R. Sutton, K.A. Sedelies, A. Ciccone, M. Jenkins, S.J. Turner, P.I. Bird, and J.A. Trapani. 2006. Cytotoxic T lymphocyte-induced killing in the absence of granzymes $\mathrm{A}$ and $\mathrm{B}$ is unique and distinct from both apoptosis and perforin-dependent lysis. J. Cell Biol. 173:133-144.

Wolters, P.J., C.T. Pham, D.J. Muilenburg, T.J. Ley, and G.H. Caughey. 2001 Dipeptidyl peptidase I is essential for activation of mast cell chymases, but not tryptases, in mice. J. Biol. Chem. 276:18551-18556. 


\section{University Library}

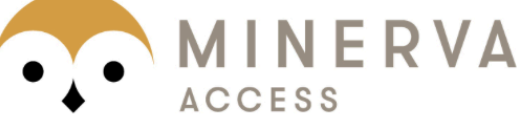

A gateway to Melbourne's research publications

Minerva Access is the Institutional Repository of The University of Melbourne

Author/s:

Sutton, VR;Waterhouse, NJ;Browne, KA;Sedelies, K;Ciccone, A;Anthony, D;Koskinen, A;Mullbacher, A;Trapani, JA

Title:

Residual active granzyme B in cathepsin C-null lymphocytes is sufficient for perforindependent target cell apoptosis

Date:

2007-02-12

Citation:

Sutton, V. R., Waterhouse, N. J., Browne, K. A., Sedelies, K., Ciccone, A., Anthony, D., Koskinen, A., Mullbacher, A. \& Trapani, J. A. (2007). Residual active granzyme B in cathepsin C-null lymphocytes is sufficient for perforin-dependent target cell apoptosis. JOURNAL OF CELL BIOLOGY, 176 (4), pp.425-433. https://doi.org/10.1083/jcb.200609077.

Persistent Link:

http://hdl.handle.net/11343/265550

License:

CC BY-NC-SA 